\title{
Imaging Findings of Bisphosphonate-Related Osteonecrosis of the Jaws: A Critical Review of the Quantitative Studies
}

\author{
André Ferreira Leite, ${ }^{1}$ Fernanda dos Santos Ogata, ${ }^{2}$ \\ Nilce Santos de Melo, ${ }^{3}$ and Paulo Tadeu de Souza Figueiredo ${ }^{1}$ \\ ${ }^{1}$ Oral Radiology, Department of Dentistry, Faculty of Health Science, University of Brasília, Campus Universitario Darcy Ribeiro, \\ Asa Norte, 70910-900 Brasília, DF, Brazil \\ ${ }^{2}$ University of Brasília, Campus Universitario Darcy Ribeiro, Asa Norte, 70910-900 Brasília, DF, Brazil \\ ${ }^{3}$ Oral Pathology, Department of Dentistry, Faculty of Health Science, University of Brasília, Campus Universitario Darcy Ribeiro, \\ Asa Norte, 70910-900 Brasília, DF, Brazil
}

Correspondence should be addressed to André Ferreira Leite; andreleite@unb.br

Received 26 March 2014; Accepted 9 May 2014; Published 11 June 2014

Academic Editor: Giuliano Ascani

Copyright (C) 2014 André Ferreira Leite et al. This is an open access article distributed under the Creative Commons Attribution License, which permits unrestricted use, distribution, and reproduction in any medium, provided the original work is properly cited.

\begin{abstract}
Objectives. This paper offers a critical review of published information on the imaging strategies used for diagnosing bisphosphonate-associated osteonecrosis of the jaw (BRONJ) in patients taking intravenous bisphosphonates, pointing at the different methodologies and results of existing literature. Methods. Electronic literature search was performed in order to identify as many quantitative studies that discussed the imaging findings of BRONJ up to February 2014. Initially, the search for articles was based on the following four types of imaging modalities for evaluating BRONJ: computed tomography, plain film radiographs, magnetic resonance imaging, and nuclear bone scanning. Results. Eleven out of the 79 initially selected articles met the inclusion criteria. Most of the selected articles were cross-sectional studies. Regarding the selected studies, 54.5\% have used plain films radiographs and 54.5\% were based on computed tomography findings. All of the selected studies showed a small number of patients and none of the selected studies have tested the accuracy of the imaging examination for evaluating BRONJ. Conclusions. This critical review showed a scarcity of quantitative studies that analyzed the typical imaging findings related to BRONJ. Further studies are necessary in order to analyze the role of different imaging techniques in the assessment of BRONJ.
\end{abstract}

\section{Introduction}

Bisphosphonates are the first line of treatment for metastatic bone cancer, osteoporosis, and Paget's disease. In the late 2003, cases of bisphosphonate-related osteonecrosis of the jaw (BRONJ) were first reported [1]. Since then, many studies have been performed in order to provide early diagnosis and better treatment for the patient once the BRONJ negatively affects their quality of life and increases morbidity. The cumulative incidence of BRONJ in patients taking intravenous bisphosphonates is significantly greater than in patients using oral bisphosphonates and varies from $0.8 \%$ to $12 \%$. The estimated risk of BRONJ for oral bisphosphonate users remains uncertain but the occurrence appears to range from 1 in 10000 to 1 in 100000 patient-years [2-4].
The American Academy of Oral and Maxillofacial Surgeons stated that, for the clinical diagnosis of BRONJ, patients need to exhibit all of the following three characteristics: (1) current or previous treatment with a bisphosphonate; (2) exposed, necrotic bone in the maxillofacial region that has persisted for more than eight weeks; and (3) no history of radiation therapy to the jaws [2].

$\mathrm{BRONJ}$ is categorized according to the clinical signs and symptoms into stage I, stage II, and stage III. Clinically, the disease appears as a nonhealing exposed bone area that can be accompanied by fistulization, purulent discharge, and pain [5]. Although imaging findings neither are considered diagnostic criteria nor have radiographic features for each stage, their findings corroborate the evaluation of the course, extent, and progression of the disease. The clinical examination does not usually show the full extent and severity of BRONJ 
sites beneath the mucosa [6]. Panoramic radiography, computed tomography (CT), magnetic resonance imaging (MRI), and scintigraphy are valuable imaging modalities that assist the clinical findings by revealing different aspects of bone involvement. Furthermore, these imaging examinations can help in the differential diagnosis of other diseases that resemble BRONJ in terms of clinical signs and symptoms [79].

Radiographic exam is additionally substantial since most patients with BRONJ are those undergoing other treatments and the imaging findings of BRONJ are not specific and can also be found in other conditions such as osteomyelitis, osteoradionecrosis, cancer metastasis, and Paget's disease [10]. The initial imaging findings in BRONJ appear to be focal medullary sclerosis with poor corticomedullary differentiation, which is clinically concomitant with the loosening of tooth. A usual sign of osteonecrosis of the jaw is the delayed socket healing after tooth extraction. In late disease, there is a sequestrum formation, fractures, and reaction, and when the maxilla is involved, there may be mucosal thickening in the adjacent sinus with fluid levels or purulent discharge [4].

Despite the lack of consensus on the radiographic evolution of BRONJ, the literature has shown through models the formation of a necrotic body or involucrum inside the trabeculae in sclerotic mandibular bone. The involucrum represents most likely dead bone, which becomes surrounded by a resorptive circumference that increases with time. Probably, this is a response by the bone cells to remove the dead bone. The involucrum follows the path of least resistance leading to an exposed sequestrum or, if the tooth is missing moves to the edentulous area, suggesting that this could be the mechanism of the formation for the clinically visible sequestrum [11].

A major challenge is the early diagnosis of BRONJ lesions, preferably when still there is no exposed bone, which allows better treatment and prevention of exposures. Therefore, studies that aimed to diagnose by imaging examinations the bone changes that precede the clinical alterations are shown to be of great value. In this regard, some authors have demonstrated the presence of regional bony sclerosis similar to cases of stages 1 to $3 \mathrm{BRONJ}$ in patients characterized as stage 0 BRONJ [12].

Several imaging features of BRONJ have been previously reported [5-22], including bone sclerosis, widening of the periodontal ligament space, cortical surface irregularities, persistent extraction sockets, bone fragmentation (sequestration), and osteolytic changes. However, the frequency and consistency of these findings and the correlation between imaging and clinical findings remain unclear. The correlation between imaging findings and the temporal development of BRONJ is also unclear. Therefore, this paper offers a critical review and analysis of published information on the imaging quantitative studies for BRONJ patients, pointing at the different methodologies and results of existing literature.

\section{Methodology}

2.1. Search Strategy. Electronic literature search was performed in order to identify as many quantitative studies as possible that analyzed the imaging findings of BRONJ up to February 2014. Databases including Pubmed/Medline, Scielo, Cochrane's Reviews, and Scopus were searched in English.

Initially, the search for articles was based on the type of imaging examination. For this purpose, the imaging modalities for evaluating BRONJ were divided into the following four groups: (1) computed tomography (CT), including both multidetector computed tomography (MDCT) and cone beam computed tomography (CBCT); (2) plain films, including panoramic and intraoral radiographs; (3) magnetic resonance imaging (MRI); (4) nuclear bone scanning, including scintigraphy, SPECT, or PET. Figure 1 shows the flow chart of the study selection procedure.

Reports of any study design (clinical trials, cohort, case-control, and cross-sectional studies) were included investigating the imaging strategies used for diagnosing bisphosphonate-associated osteonecrosis of the jaw in patients taking intravenous bisphosphonates. All studies that performed quantitative analyses were included. The final selection was completed after eliminating the duplicated articles, case reports, case series, reviews of the literature, editorials, anecdotal letters, letters to the editors, and those articles that were not related to imaging findings for evaluating BRONJ patients.

2.2. Statistical Analysis. Statistical analysis of data from the selected studies was not attempted due to the variations in the study design, methodology, and choice of imaging modality.

\section{Results}

From the initial search, most of the excluded articles were not related to imaging findings of BRONJ. After eliminating the duplicated articles and those that were not related to imaging findings of BRONJ, the initial database search yielded 79 different abstracts. Nevertheless, only eleven of these initially selected studies met the inclusion criteria $[6,11,13-21]$.

Regarding the excluded articles from the second search, most of the studies were case series/reports of cases $(63.3 \%)$ that only described imaging features of BRONJ patients. Figures 2 and 3 show examples of the main imaging findings of BRONJ in two patients taking intravenous zoledronic acid. Furthermore, five excluded studies were performed in animals (6.3\%) and 30.4\% were reviews of the literature.

3.1. Characteristics of Included Papers. Information on the study patient's demographics, study design, imaging modalities, and technical parameters of the eleven included papers is outlined in Table 1. Table 2 shows the objectives, main results, and main conclusions of each selected studies.

Regarding the selected studies, 54.5\% (6 studies) have used plain films radiographs and 54.5\% (6 studies) were based on computed tomography findings. Only two quantitative studies were found with MRI (18.2\%) and with nuclear bone scanning $(18.2 \%)$. 


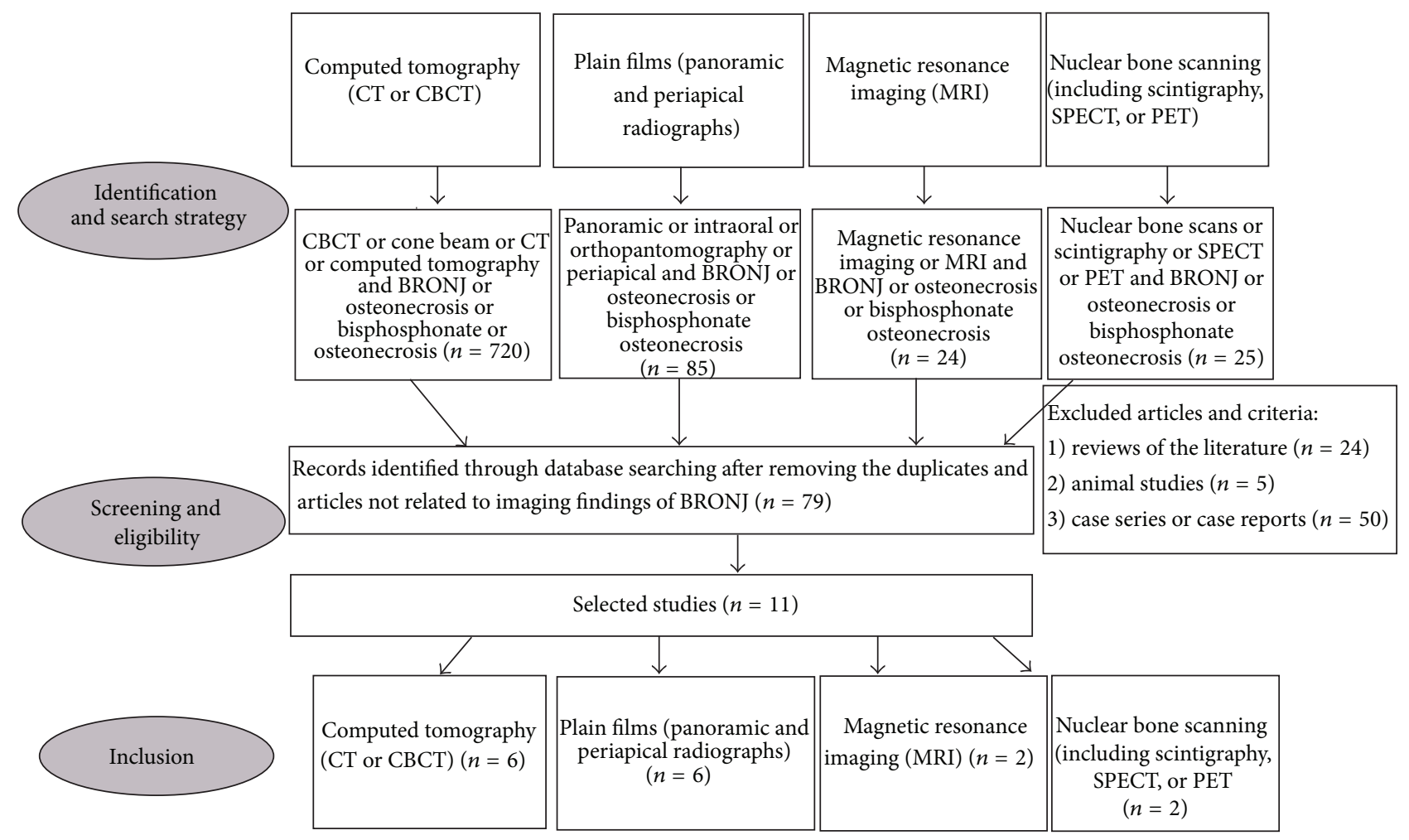

FIGURE 1: Flow chart of the study selection procedure.

\section{Discussion}

As far as we know, the present study is the first critical review aiming at discussing little evidence about imaging findings of BRONJ. Initially, we intended to perform a meta-analysis of the existing literature regarding imaging modalities for BRONJ patients. However, due to the scarcity of quantitative studies with a similar methodology, it was only possible to perform a critical review and qualitative analysis of the published studies related to this issue.

In our review, many studies (63.3\%) were retrospective case series or case reports with unclear incidences and frequency estimates of imaging findings. For this reason, this kind of studies entered in the exclusion criteria of our review. An attempt has been made to collate, compare, and discuss the methodology and results of different studies that quantitatively evaluated the imaging findings of BRONJ in patients taking intravenous bisphosphonates. The reading of these selected studies showed a significant heterogeneity. In addition to the small amount of selected articles, the comparison of the findings was difficult due to the significant methodological differences between each study, conflicting results, small sample sizes, and the variability of imaging techniques. Furthermore, the absence of diagnostic test studies that report the specificity and sensitivity precluded the analysis of accuracy of each imaging modality.

Few studies have evaluated imaging findings in bisphosphonate-treated patients with stage 0 disease in the absence of bone exposure [11, 12]. The former was a prospective study conducted with clinical and dental panoramic analysis of 60 patients. Of these 60 patients, thirty were treated with zolendronate and 30 composed the control group. Patients treated with the intravenous aminobisphosphonate presented a statistically significant increase in the number of radiographic abnormalities compared with the control group. However, this selected study has not described or discussed the radiographic findings. The second aforementioned study analyzed patients receiving oral bisphosphonate therapy which is not the main risk group for developing BRONJ. As this study was only descriptive, it was excluded from our sample.

Diagnosis of BRONJ is usually made at the late stage when there is bone exposure to the oral cavity. Standard diagnosis based on clinicoradiological criteria is still lacking and there are no clinicoradiological guidelines for the health professionals to follow. In our systematic review, four of the eleven selected studies have used exclusively plain films such as panoramic and periapical radiographs [11, 14, 17, 20]. However, these studies have different objectives and methodologies and different patient populations and types of bisphosphonate therapies, which preclude a direct comparison of their results. Some authors have stated that a higher risk of developing BRONJ apparently may be predicted detecting the rise of alveolar bone mineral density that frequently occurs near the necrotic lesion [17] and by the presence of a radiographic periodontal ligament widening [14].

Dental panoramic radiograph and computed tomography can be considered as the most widely available imaging techniques for BRONJ evaluation. This can explain why most of the selected studies have used those imaging modalities [11, 


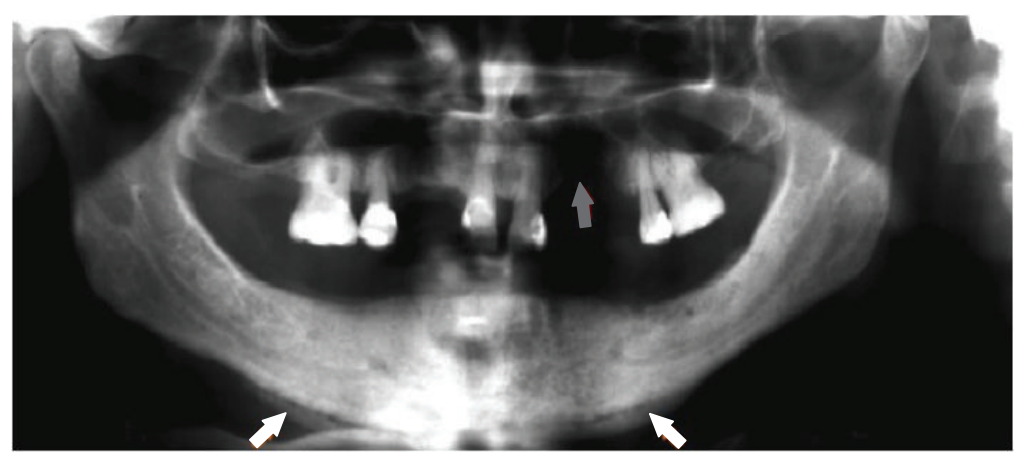

(a)

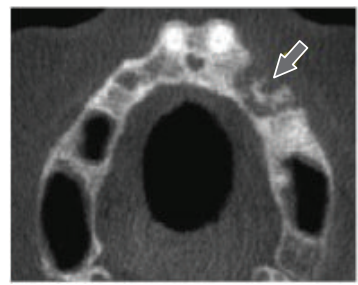

(b)

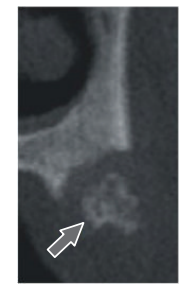

(c)

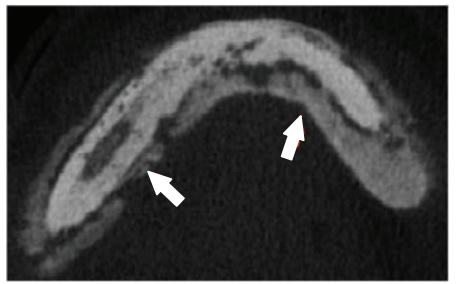

(d)

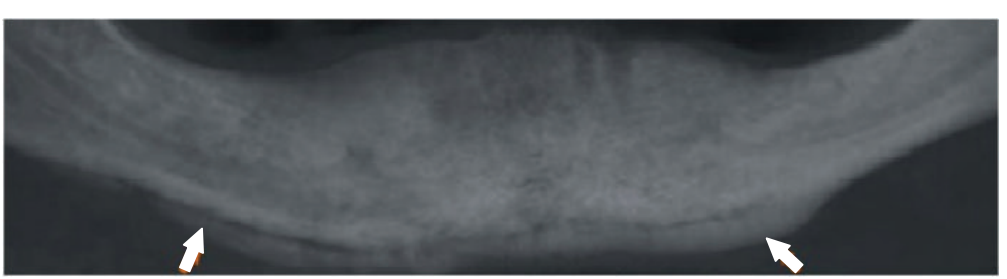

(e)

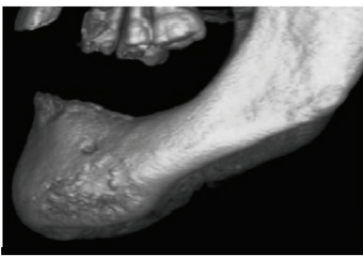

(g)

FIGURE 2: Imaging findings of a 57-year-old woman with metastatic breast carcinoma receiving intravenous zoledronic acid. (a) Panoramic radiograph showing maxillary involvement with radiographic evidence of osteolysis (gray arrow). (b) and (c) axial and cross-sectional CBCT views, respectively, showing the necrotic area with bone sequestrum in the left maxilla (gray arrow). (d) Axial CBCT image showing the extent of mandible bone involvement with periosteal bone reaction. The periosteal bone reaction changed the mandibular morphology, as it can be seen in the two-dimensional multiplanar reconstruction image ((e), white arrow) and in the 3D images (frontal view (f) and sagittal view $(\mathrm{g}))$.

13-16, 18-20]. Furthermore, they usually detect dentoosseous changes related to this entity, including bone sclerosis, cortical surface irregularities, persistent extraction sockets, bone fragmentation (sequestration), and osteolysis.

Despite being the most used imaging modalities for BRONJ evaluation, there are some contradictory results on the selected studies. Some authors have suggested that panoramic radiographs are useful for evaluating BRONJ [11, 20]. On the other hand, other authors have stated that these radiographs are of limited value for this purpose $[13,16]$. The differences may be related to the imaging modalities used in the studies. The selected studies that emphasized the role of the plain film radiographs for BRONJ evaluation have not used 3D images $[11,14,17,20]$. On the other hand, the criticism of some authors regarding plain film radiographs was based on comparison with other $3 \mathrm{D}$ imaging modalities such as CT and MRI $[16,20]$. Panoramic radiograph may be a useful and readily accessible imaging examination for the initial radiologic investigation in patients treated with intravenous bisphosphonates. This kind of radiography allows quick visualization of the entire affected area and seems to be able to demonstrate clear signs of osteolytic lesions mainly when radiopaque sequestra are present or when osteolysis is combined with osteosclerosis [5, 7]. In a previous cross section study with 39 patients, a correlation was found between focal panoramic radiographic findings of bone sclerosis and surface irregularity with clinical sites of BRONJ [20]. However, the disadvantages of panoramic radiograph should be recognized, such as missing definition among the margins of the necrotic areas and healthy bone, the difficulty in distinguishing osteonecrosis of a malignant lesion when an osteolytic lesion is present, and the limited image in a two-dimensional view of three-dimensional structures. Such limitations restrict the understanding of all the extent of the lesion [5, 14]. As a conventional radiograph, panoramic images often suffer from magnification, distortion, and superimposition. Moreover, a successful panoramic radiograph requires careful positioning of the patient and proper technique. Therefore, the limitations of this imaging modality for BRONJ patients should be 


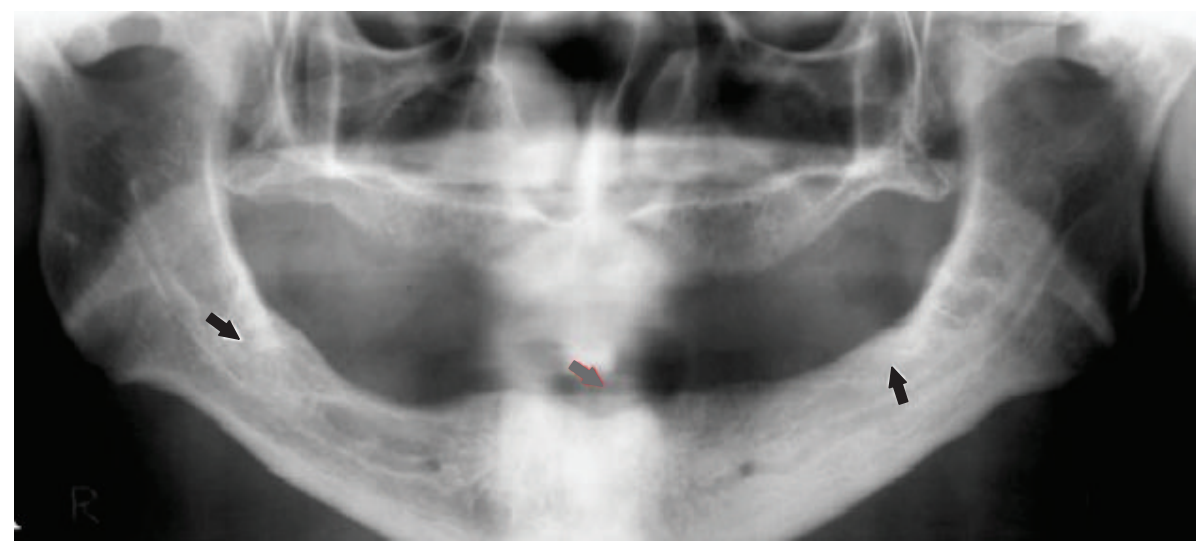

(a)

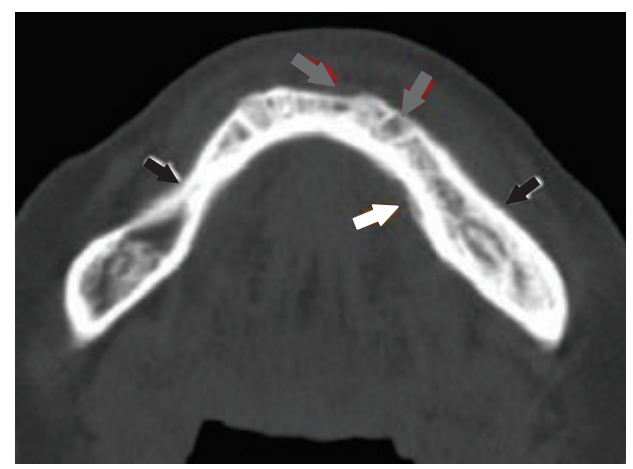

(b)

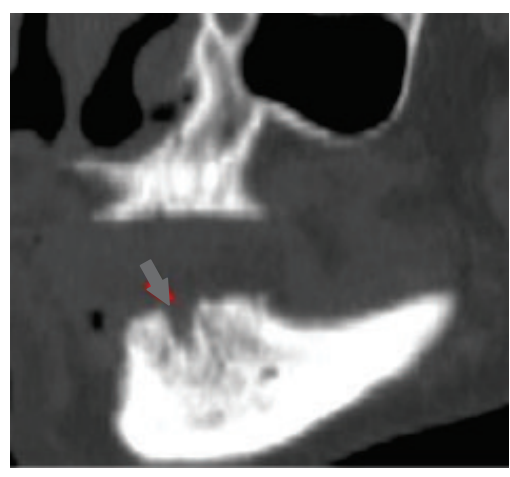

(c)

FIGURE 3: Imaging findings of a 65-year-old woman with metastatic breast carcinoma receiving intravenous zoledronic acid. (a) Panoramic radiograph showing an osteolytic lesion in the anterior mandible (gray arrow) and areas of osteosclerosis in the posterior regions (black arrows). (b) Axial CBCT image reveals areas of osteolysis (gray arrows), areas of osteosclerosis (black arrows), and a periosteal bone reaction in the left mandible (white arrow). (c) Sagittal CBCT image demonstrates a nonhealing extraction socket in the anterior mandible.

emphasized, especially in elderly or noncollaborating patients $[23,24]$.

Computed tomography (including multidetector CT or СВCT) has been demonstrated to be superior to panoramic in detection and evaluation of BRONJ, particularly with regard to soft tissue swelling, new bone, and sequestrum $[13,16]$. CBCT may also be used for detection of bone alterations by evaluating the fractal dimension of the alveolar process [18] and measuring the mandibular cortical bone that are higher in BRONJ patients [19]. CBCT may also allow the detection of subclinical, small involucra and has potential in monitoring the progression of the lesions [25]. Compared with multidetector CT, CBCT is easy to use, with short acquisition scan times and high resolution, can be performed while patients are in the upright position, and is of low cost [26].

Our systematic review has shown that the selected studies have used different imaging modalities such as periapical radiographs [14, 17], panoramic radiographs $[11,13,14,16$, $20]$, multidetector computed tomography $[13,16]$, cone beam computed tomography $[15,18,20]$, MRI $[15,16]$, PET/CT [15], and scintigraphy and SPECT [21]. Apparently, CT scan is extremely useful in defining the features and extent of the lesions and, in selected cases, an MRI can add value to the radiological findings by showing the soft tissue involvement. However, there have been no studies that have rigorously compared these various modalities for their utility in evaluating BRONJ, especially regarding clinically relevant end points [22].

Although imaging examination can be very useful in determining the extent of bony changes, only one selected study has compared different imaging modalities for this purpose [15]. PET/CT and MRI revealed more extensive involvement of BRONJ compared with CBCT and clinical examinations. However, only 10 patients have been evaluated in this prospective cross-sectional study. Further prospective studies are necessary to verify which imaging modality is better for evaluating the extent of BRONJ. The role of nuclear bone scanning for evaluating patients taking intravenous bisphosphonates also deserves further investigation. In a cohort study with 22 subjects, some authors have demonstrated that the relative quantification of tracer uptake provides prognostic information independent of clinical stage of BRONJ [21]. Although scintigraphy is a very sensitive investigation it may be used as a screening test to detect subclinical osteonecrosis in patients receiving bisphosphonates $[7,27]$, but it should be kept in mind that the rate of false positives may be high due to the lack of specificity [28]. 


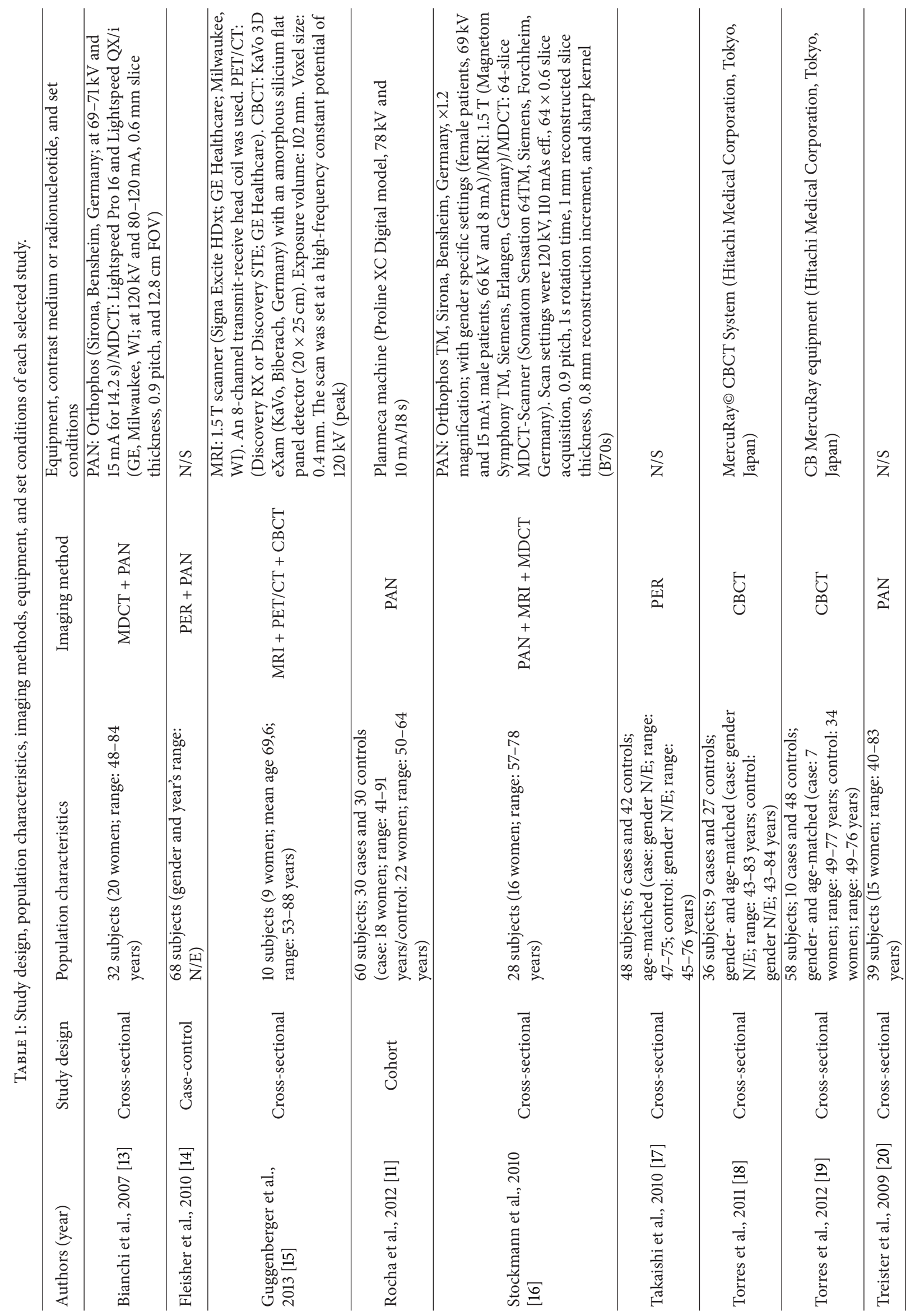




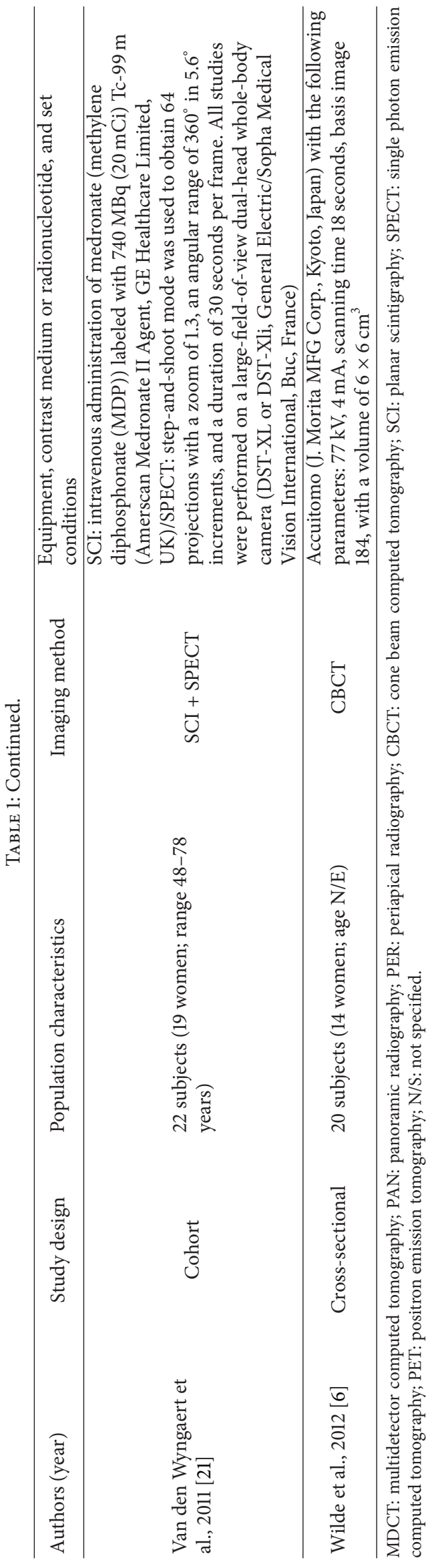




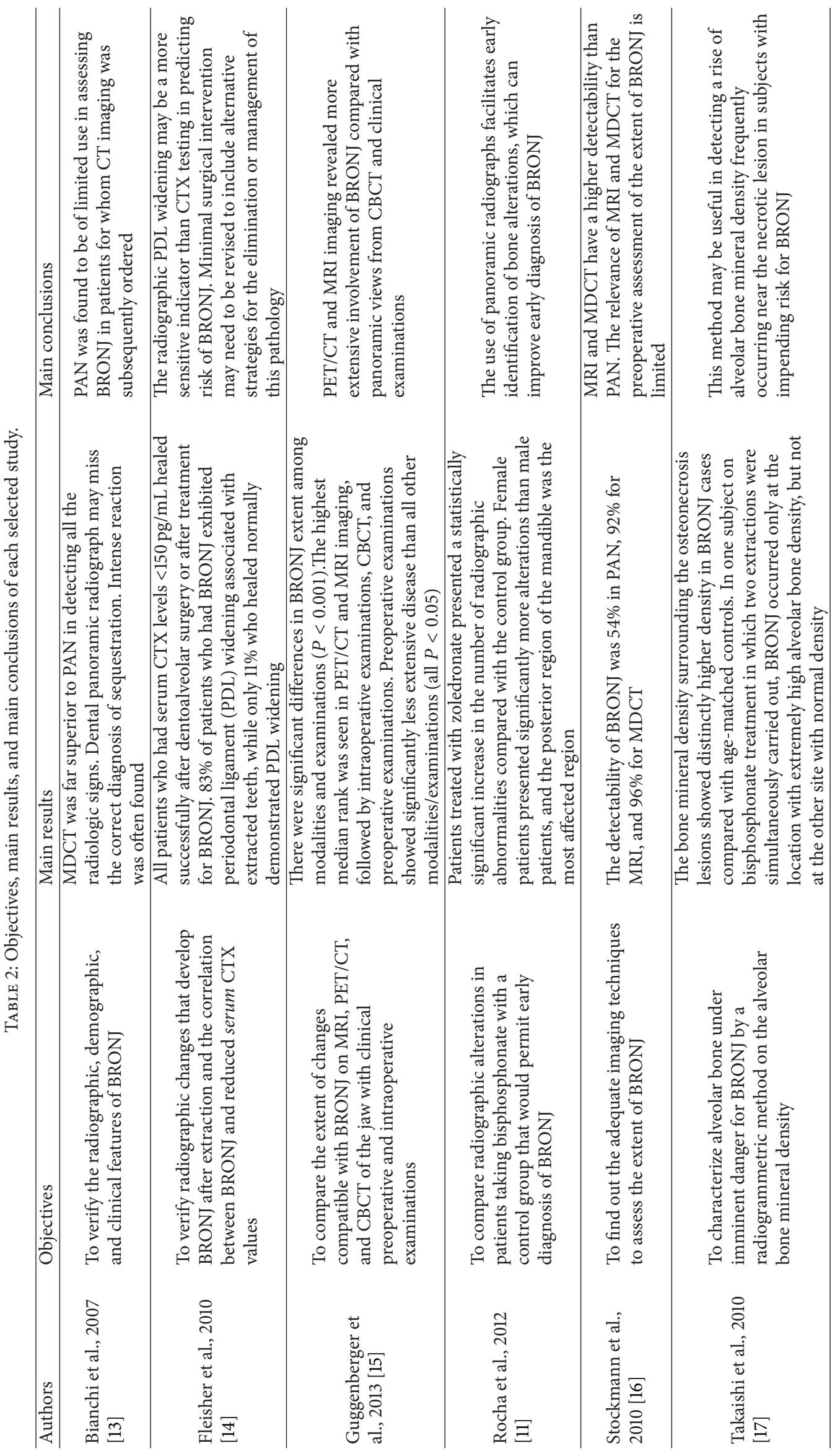




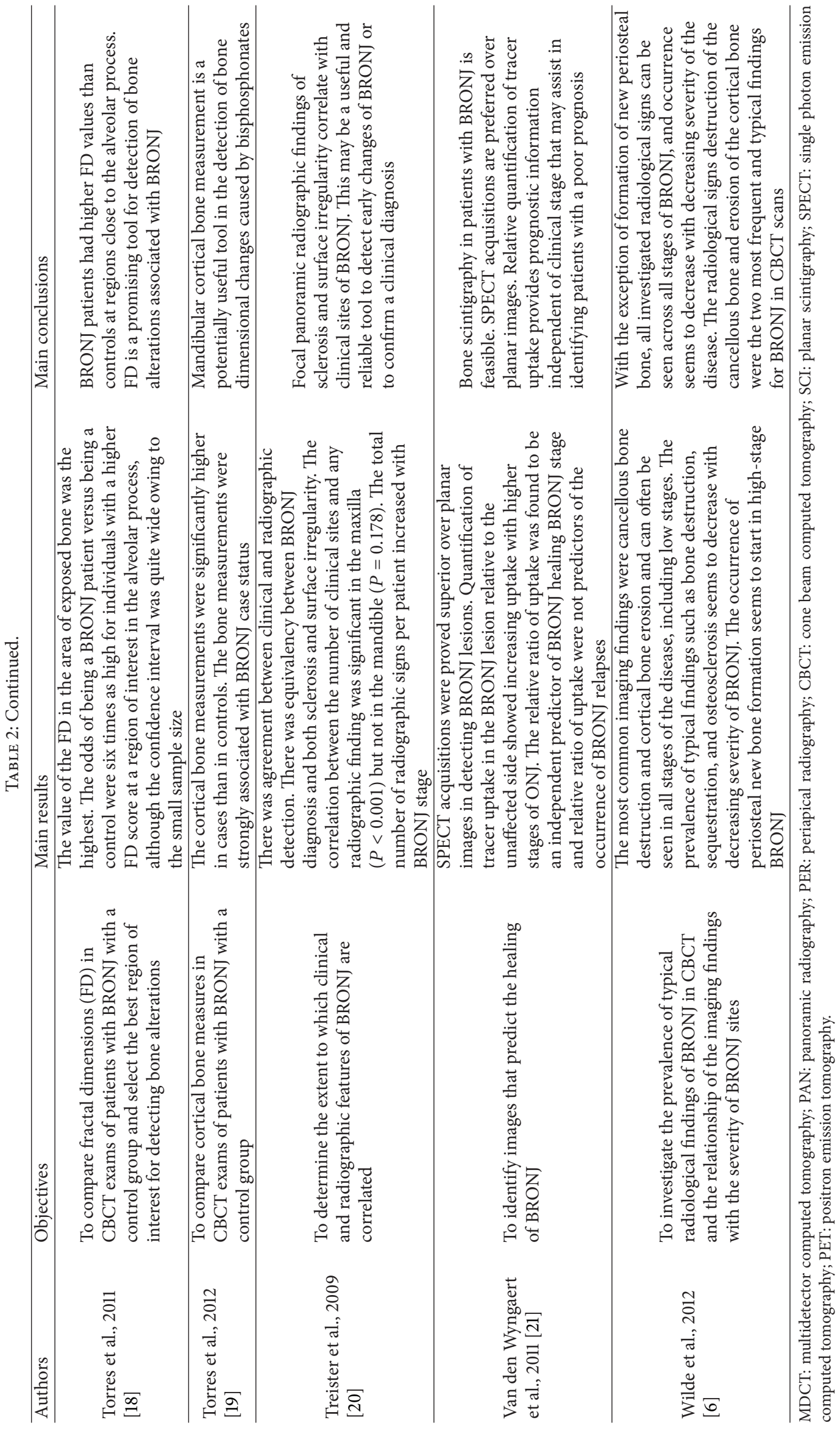


This study has its own limitations. Due to the scarcity of the literature it was not possible to compare quantitatively the selected studies. Consequently, it was decided to select all the quantitative studies, despite of the significant differences in methodologies, imaging modalities, kind of studies, and populations. Although several theories about the etiology of the BRONJ have been advanced, many questions remain unanswered, especially regarding the pathophysiology [3]. The complete understanding of the pathogenesis may also contribute to the development of prevention and treatment guidelines, including the guidelines for prescription of imaging examinations.

In conclusion, this critical review showed a scarcity of quantitative studies that analyzed the typical imaging findings related to BRONJ. Further studies are necessary in order to analyze the frequency and how the typical findings appear, and also the timing of their appearance. Clinical guidelines for BRONJ need to include which imaging modality should be performed for BRONJ patients and at what time intervals. Although conventional radiographs can demonstrate evidence of BRONJ, especially when disease is advanced, there are limitations of these imaging modalities, regarding their $2 \mathrm{D}$ nature and also the technical characteristics. While CBCT scans provide more information regarding the extent of bone changes, the usefulness of this imaging modality in asymptomatic individuals should be better investigated. Further study would be useful to identify, based on clinical and radiographic factors, whether CBCT examinations are justified for all BRONJ patients. Nuclear medicine modalities, such as PET/CT, may also be considered as promising tools for BRONJ evaluation. Diagnostic test studies and the comparison of the various imaging modalities are still necessary.

\section{Conflict of Interests}

The authors declare that there is no conflict of interests regarding the publication of this paper.

\section{Acknowledgment}

This paper was supported by DPP/UnB.

\section{References}

[1] R. E. Marx, "Pamidronate (Aredia) and zoledronate (Zometa) induced avascular necrosis of the jaws: a growing epidemic," Journal of Oral and Maxillofacial Surgery, vol. 61, no. 9, pp. 11151117, 2003.

[2] S. L. Ruggiero, T. B. Dodson, L. A. Assael, R. Landesberg, R. E. Marx, and B. Mehrotra, "American association of oral and maxillofacial surgeons position paper on bisphosphonaterelated osteonecrosis of the jaw," Australian Endodontic Journal, vol. 35, no. 3, pp. 119-130, 2009.

[3] S. L. Silverman and R. Landesberg, "Osteonecrosis of the jaw and the role of bisphosphonates: a critical review," American Journal of Medicine, vol. 122, no. 2, pp. S33-S45, 2009.

[4] A. E. Haworth and J. Webb, "Skeletal complications of bisphosphonate use: what the radiologist should know," British Journal of Radiology, vol. 85, no. 1018, pp. 1333-1342, 2012.
[5] S. Chiandussi, M. Biasotto, F. Dore, F. Cavalli, M. A. Cova, and R. Di Lenarda, "Clinical and diagnostic imaging of bisphosphonate-associated osteonecrosis of the jaws," Dentomaxillofacial Radiology, vol. 35, no. 4, pp. 236-243, 2006.

[6] F. Wilde, M. Heufelder, K. Lorenz et al., "Prevalence of cone beam computed tomography imaging findings according to the clinical stage of bisphosphonate-related osteonecrosis of the jaw," Oral Surgery, Oral Medicine, Oral Pathology and Oral Radiology, vol. 114, no. 6, pp. 804-811, 2012.

[7] K. Arce, L. A. Assael, J. L. Weissman, and M. R. Markiewicz, "Imaging findings in bisphosphonate-related osteonecrosis of jaws," Journal of Oral and Maxillofacial Surgery, vol. 67, no. 5, pp. 75-84, 2009.

[8] A. Bedogni, S. Blandamura, Z. Lokmic et al., "Bisphosphonateassociated jawbone osteonecrosis: a correlation between imaging techniques and histopathology," Oral Surgery, Oral Medicine, Oral Pathology, Oral Radiology and Endodontology, vol. 105, no. 3, pp. 358-364, 2008.

[9] Y. Morag, M. Morag-Hezroni, D. A. Jamadar et al., "Bisphosphonaterelated osteonecrosis of the jaw: a pictorial review," Radiographics, vol. 29, no. 7, pp. 1971-1984, 2009.

[10] G. M. Fatterpekar, J. V. Emmrich, J. A. Eloy, and A. Aggarwal, "Bone-within-bone appearance: a red flag for biphosphonateassociated osteonecrosis of the jaw," Journal of Computer Assisted Tomography, vol. 35, no. 5, pp. 553-556, 2011.

[11] G. C. M. A. Rocha, G. C. Jaguar, C. R. Moreira, E. G. Neves, F. P. Fonseca, and E. N. Pedreira, "Radiographic evaluation of maxillofacial region in oncology patients treated with bisphosphonates," Oral Surgery, Oral Medicine, Oral Pathology and Oral Radiology, vol. 114, no. 5, pp. S19-S25, 2012.

[12] M. Hutchinson, F. O'Ryan, V. Chavez et al., "Radiographic findings in bisphosphonate-treated patients with stage 0 disease in the absence of bone exposure," Journal of Oral and Maxillofacial Surgery, vol. 68, no. 9, pp. 2232-2240, 2010.

[13] S. D. Bianchi, M. Scoletta, F. B. Cassione, G. Migliaretti, and M. Mozzati, "Computerized tomographic findings in bisphosphonate-associated osteonecrosis of the jaw in patients with cancer," Oral Surgery, Oral Medicine, Oral Pathology, Oral Radiology and Endodontology, vol. 104, no. 2, pp. 249-258, 2007.

[14] K. E. Fleisher, G. Welch, S. Kottal, R. G. Craig, D. Saxena, and R. S. Glickman, "Predicting risk for bisphosphonate-related osteonecrosis of the jaws: CTX versus radiographic markers," Oral Surgery, Oral Medicine, Oral Pathology, Oral Radiology and Endodontology, vol. 110, no. 4, pp. 509-516, 2010.

[15] R. Guggenberger, D. R. Fischer, P. Metzler et al., "Bisphosphonate-induced osteonecrosis of the jaw: comparison of disease extent on contrast-enhanced MR imaging, [18F] fluoride $\mathrm{PET} / \mathrm{CT}$, and conebeam CT imaging," American Journal of Neuroradiology, vol. 34, no. 6, pp. 1242-1247, 2013.

[16] P. Stockmann, F. M. Hinkmann, M. M. Lell et al., "Panoramic radiograph, computed tomography or magnetic resonance imaging. Which imaging technique should be preferred in bisphosphonate-associated osteonecrosis of the jaw? A prospective clinical study," Clinical Oral Investigations, vol. 14, no. 3, pp. 311-317, 2010

[17] Y. Takaishi, T. Ikeo, M. Nakajima, T. Miki, and T. Fujita, "A pilot case-control study on the alveolar bone density measurement in risk assessment for bisphosphonate-related osteonecrosis of the jaw," Osteoporosis International, vol. 21, no. 5, pp. 815-825, 2010.

[18] S. R. Torres, C. S. K. Chen, B. G. Leroux, P. P. Lee, L. G. Hollender, and M. M. Schubert, "Fractal dimension evaluation of cone 
beam computed tomography in patients with bisphosphonateassociated osteonecrosis," Dentomaxillofacial Radiology, vol. 40, no. 8, pp. 501-505, 2011.

[19] S. R. Torres, C. S. K. Chen, B. G. Leroux et al., "Mandibular cortical bone evaluation on cone beam computed tomography images of patients with bisphosphonate-related osteonecrosis of the jaw," Oral Surgery, Oral Medicine, Oral Pathology and Oral Radiology, vol. 113, no. 5, pp. 695-703, 2012.

[20] N. Treister, N. Sheehy, E. H. Bae, B. Friedland, M. Lerman, and S. Woo, "Dental panoramic radiographic evaluation in bisphosphonate-associated osteonecrosis of the jaws," Oral Diseases, vol. 15, no. 1, pp. 88-92, 2009.

[21] T. van den Wyngaert, M. T. Huizing, E. Fossion, and J. B. Vermorken, "Prognostic value of bone scintigraphy in cancer patients with osteonecrosis of the jaw," Clinical Nuclear Medicine, vol. 36, no. 1, pp. 17-20, 2011.

[22] N. S. Treister, B. Friedland, and S.-B. Woo, "Use of cone-beam computerized tomography for evaluation of bisphosphonateassociated osteonecrosis of the jaws," Oral Surgery, Oral Medicine, Oral Pathology, Oral Radiology and Endodontology, vol. 109, no. 5, pp. 753-764, 2010.

[23] F. Kaviani, M. Johari, and F. Esmaeili, "Evaluation of common errors of panoramic radiographs in tabriz faculty of dentistry," Journal of Dental Research, Dental Clinics, Dental Prospects, vol. 2, pp. 99-101, 2008.

[24] R. H. Rondon, Y. C. Pereira, and G. C. do Nascimento, "Common positioning errors in panoramic radiography: a review," Imaging Science in Dentistry, vol. 44, pp. 1-6, 2014.

[25] C. Barragan-Adjemian, L. Lausten, D. B. Ang, M. Johnson, J. Katz, and L. F. Bonewald, "Bisphosphonate-related osteonecrosis of the jaw: model and diagnosis with cone beam computerized tomography," Cells Tissues Organs, vol. 189, no. 1-4, pp. 284-288, 2009.

[26] F. A. Quereshy, T. A. Savell, and J. M. Palomo, "Applications of cone beam computed tomography in the practice of oral and maxillofacial surgery," Journal of Oral and Maxillofacial Surgery, vol. 66, no. 4, pp. 791-796, 2008.

[27] F. S. O'Ryan, S. Khoury, W. Liao et al., "Intravenous bisphosphonate-related osteonecrosis of the jaw: bone scintigraphy as an early indicator," Journal of Oral and Maxillofacial Surgery, vol. 67, no. 7, pp. 1363-1372, 2009.

[28] F. Dore, L. Filippi, M. Biasotto, S. Chiandussi, F. Cavalli, and R. Di Lenarda, "Bone scintigraphy and SPECT/CT of bisphosphonate-induced osteonecrosis of the jaw," Journal of Nuclear Medicine, vol. 50, no. 1, pp. 30-35, 2009. 


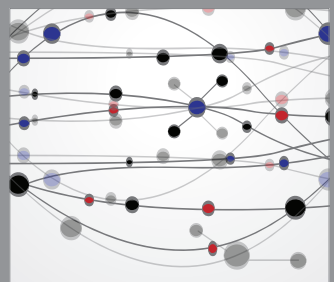

The Scientific World Journal
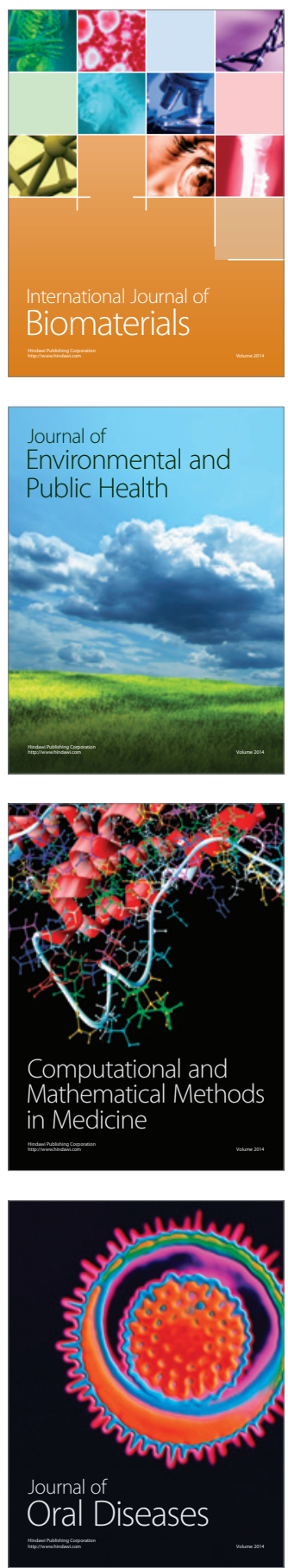
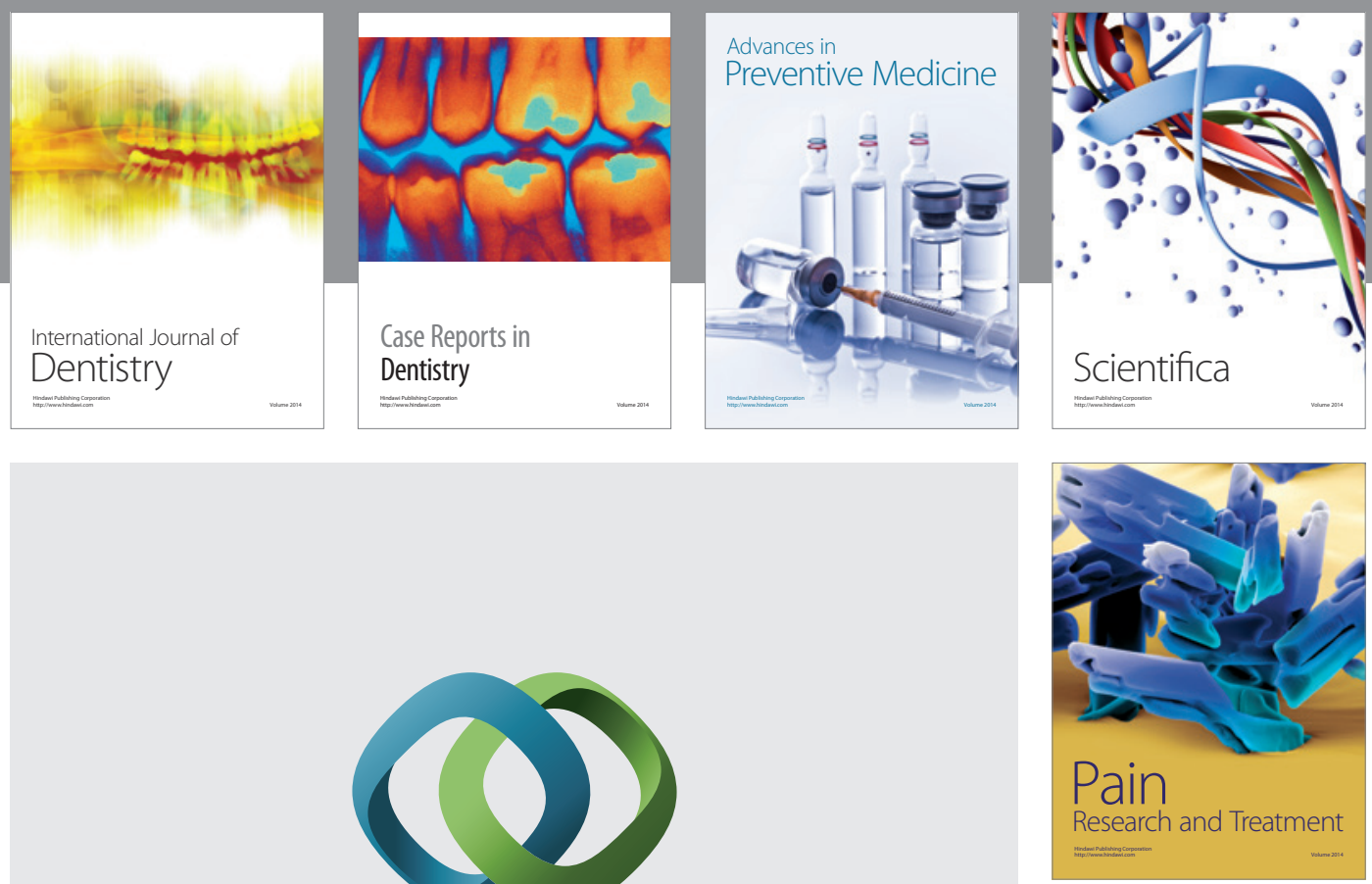

\section{Hindawi}

Submit your manuscripts at

http://www.hindawi.com
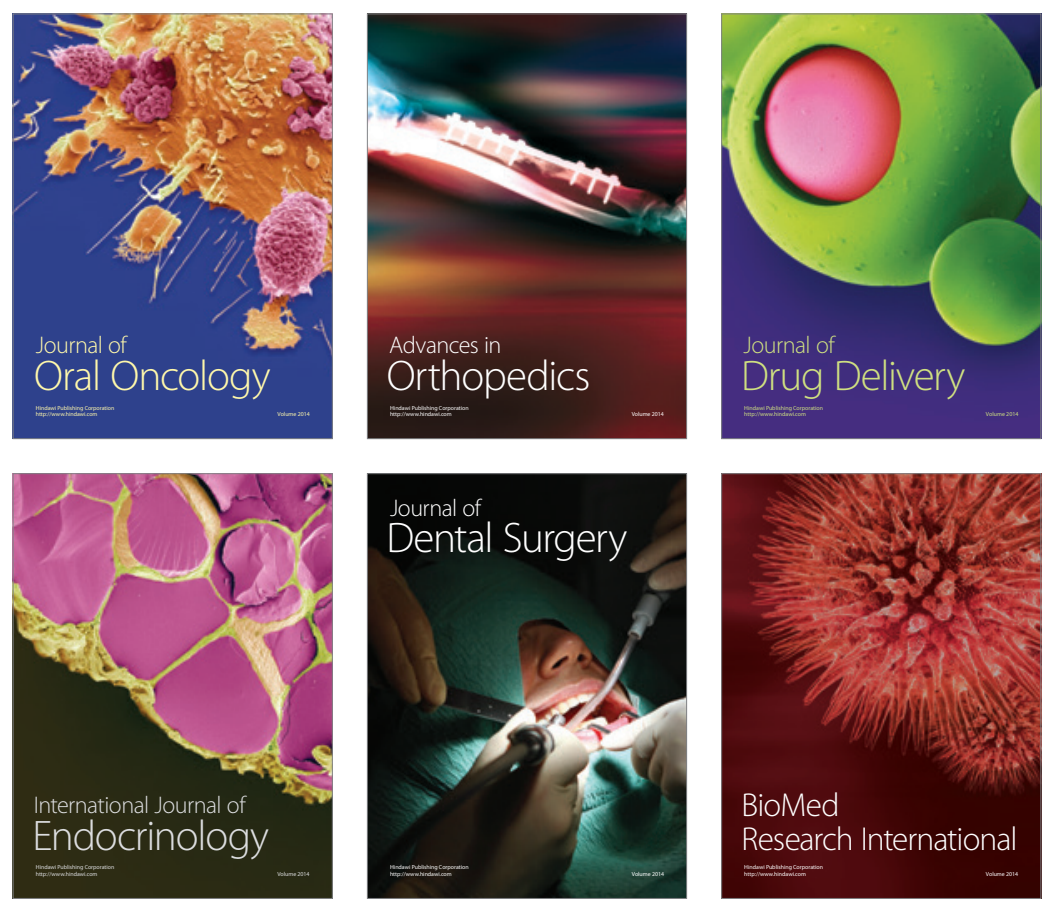

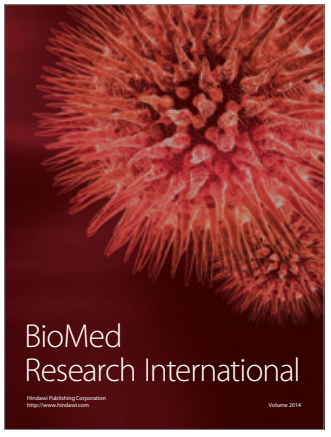

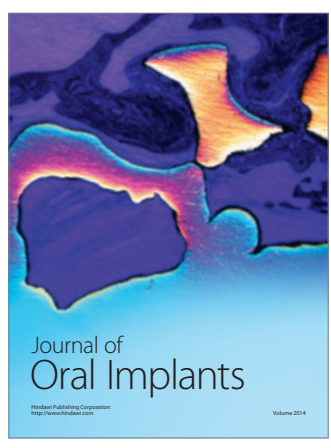
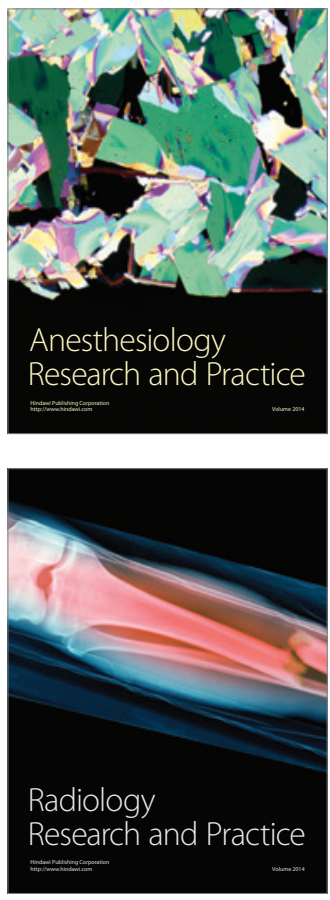